\title{
Arsénico edáfico y su distribución en el distrito de riego 017: uso de métodos de interpolación
}

\section{Edaphic arsenic and its distribution in irrigation district 017: using interpolation methods}

\author{
Norma E. Rodríguez Garrido ${ }^{1}$, Miguel Ángel Segura Castruita ${ }^{2 *}$, \\ Jorge A. Orozco Vidal'2, Manuel Fortis Hernández², Pablo Preciado Rangel², \\ Jesús Olague Ramírez ${ }^{2}$ y Pablo Yescas Coronado
}

\footnotetext{
${ }^{1}$ Instituto Tecnológico de Torreón, Estudiante de Maestría, ${ }^{2}$ Profesor Investigador DEPI. Carretera Torreón-San Pedro Km 7.5, Ejido Ana. 27170 Torreón, Coahuila, México.

‡Autor responsable (dmilys5@hotmail.com)
}

\section{RESUMEN}

Los constituyentes del suelo (arcillas, carbonatos, materia orgánica y óxidos e hidróxidos), intervienen en la movilidad del arsénico (As) y determinan su variación espacial; por lo cual, su cartografía puede contribuir al entendimiento de la presencia de este metaloide en el suelo. Los objetivos de esta investigación fueron determinar la concentración de As en los suelos del Distrito de Riego 017 (DR017) de México y establecer su distribución espacial, utilizando dos métodos de interpolación. Cuatro etapas fueron realizadas; la primera consistió en trabajo de gabinete y campo, en la que 33 sitios de muestreo fueron localizados en un polígono del DR017, posteriormente en cada sitio, muestras simples de suelo $(0-30 \mathrm{~cm}$ de profundidad) fueron colectadas. La segunda y tercera se llevaron a cabo en laboratorio, en las cuales, a cada muestra se determinó la concentración de As disponible, contenidos de arcilla, carbonatos de calcio y fósforo disponible y su regresión. La cuarta etapa tuvo dos partes, la elaboración de dos mapas con dos métodos de interpolación (Interpolador Ponderación Inversa de la Distancia, IDW en sus siglas en inglés y Kriging ordinario, $\mathrm{KO}$ ), así como la verificación de la precisión de cada mapa. Los resultados mostraron que, la distribución del As en el DR017 no es uniforme y está asociada con el contenido de arcilla y $\mathrm{CaCO}_{3}$. De tal manera que, las concentraciones de As fluctuaron de 0.07 a $1.89 \mathrm{mg} \mathrm{kg}^{-1}$, donde las mayores concentraciones de este metaloide (1.39-1.89 $\left.\mathrm{mg} \mathrm{kg}^{-1}\right)$ se relacionaron positivamente $\left(\mathrm{R}^{2}=0.9058\right)$ con altos contenidos de arcilla (39.44 a 43.44\%). El mapa de distribución espacial de As obtenido con KO, tuvo

Cita recomendada:

Rodríguez Garrido, N. E., M. Á. Segura Castruita, J. A. Orozco Vidal, M. Fortis Hernández, P. Preciado Rangel, J. Olague Ramírez y P. Yescas Coronado. 2017. Arsénico edáfico y su distribución en el distrito de riego 017: uso de métodos de interpolación. Terra Latinomericana 35: 19-28. la mayor precisión $(75.7 \%)$, en el cual se muestra que la concentración aumenta en un sentido sur-norte, donde el área de mayor concentración está en la parte norte del distrito.

Palabras clave: arsenatos, kriging, IDW, geoestadístico.

\section{SUMMARY}

Soil constituents (clays, carbonates, organic matter and oxides and hydroxides), involved in the mobility of arsenic (As), determine its spatial variation; thus, the mapping can contribute to the understanding of the presence of this metalloid on the soil. The objectives of this research were to determine the concentration of As in soils of Irrigation District 017 (DR017) of Mexico and establish its spatial distribution using two interpolation methods. Four stages were performed; the first consisted of desk work and field, in which 33 sampling sites were located in a polygon of DR017, then at each site, simple soil samples (0-30 cm depth) were collected. The second and third were carried out in laboratory, in which, in each sample the concentration of As available, clay content, carbonates of calcium and phosphorus available and their regression were determined. The fourth stage had two parts, the development of two maps with two interpolation methods (Interpolator Weighting Inverse Distance, IDW its acronym in English and ordinary Kriging, $\mathrm{OK}$ ) and verification of the accuracy of each map. The results showed that the distribution of As in the DR017 is not uniform and is associated with $\mathrm{CaCO}_{3}$ and clay content. So that, As concentrations ranged from 0.07 to $1.89 \mathrm{mg} \mathrm{kg}^{-1}$, where the highest concentrations of 
this metalloid (1.39-1.89 $\mathrm{mg} \mathrm{kg}^{-1}$ ) were positively correlated $\left(\mathrm{R}^{2}=0.9058\right)$ with high content of clay (39.44 to $43.44 \%)$. The map of spatial distribution of As obtained by KO, had the highest accuracy $(75.7 \%)$, which shows that the concentration increases in a north-south direction, where the area of greatest concentration is in the northern part of the district.

Index words: arsenates, soil, Kriging, IDW, geostatistical.

\section{INTRODUCCIÓN}

El suelo como cuerpo tridimensional, viviente y dinámico, es parte integral del ecosistema terrestre, varía gradualmente en el espacio geográfico y a través del tiempo, forma complejos patrones por la interrelación de múltiples factores ambientales y funciones naturales forzadas (Segura-Castruita, 2014). La planeación para el uso racional de este recurso, requiere del conocimiento de su variabilidad espacial y sus características físicas y químicas (Donghee et al., 2012; Kolat et al., 2012). La evaluación del estado actual del recurso edáfico en una región agrícola es de importancia para su uso, manejo y conservación (Flores et al., 2002; Anguiano et al., 2003).

El Distrito de Riego 017 (DR017) en la Región Lagunera, entre los estados de Coahuila y Durango, México, tiene problemas de arsenicismo (Castruita et al., 2011); de tal manera que en algunos suelos (grupo de los Fluvisoles) de esta región hay concentraciones de arsénico (As) disponible que fluctúan de 0.648 a $1.23 \mathrm{cmol}(+) \mathrm{kg}^{-1}$, valores que no representan un problema de contaminación natural por arsenicismo para los suelos agrícolas de la región (Hernández et al., 2013). Alam et al. (2007) indican que la presencia y acumulación de este elemento en el suelo es resultado de procesos geoquímicos y de actividades antropogénicas. El arsénico en el suelo puede permanecer en dos estados de oxidación, como arsenitos $\left(\mathrm{AsO}_{3}\right)$ o como arsenatos $\left(\mathrm{AsO}_{4}\right)$; siendo este último estado el más habitual en condiciones de campo y laboratorio, y el más susceptible de ser adsorbido por las partículas del suelo (Violante y Pigna, 2002). Los constituyentes del suelo (arcillas silicatadas, amorfas, carbonatos y óxidos e hidróxidos de hierro o aluminio), juegan un papel importante en la movilidad del arsénico dentro de éste (Bissen y Frimmel, 2003; Zhao et al., 2009) y determinan la compleja variación espacial de la concentración del
As en el suelo (Lin, 2008). Otro factor que puede influir en la concentración de arsénico en el suelo, es la adición de fósforo en forma de fertilizante a través de la actividad agrícola (Fitz y Wenzel, 2002). En la Región Lagunera, Hernández et al. (2013) concluyeron que la adición de fósforo al suelo da como resultado el incremento de la concentración de As en la solución del suelo hasta en 662\%; asimismo, destacan la necesidad de establecer la distribución espacial de este elemento, para un mejor entendimiento de la presencia de arsénico en los suelos de la región. La utilidad de la geoestadística y sistemas de información geográficos (SIG) en el establecimiento de la distribución espacial de contaminantes del suelo ha sido demostrada; de tal manera que, la cartografía de la distribución de As puede contribuir en el entendimiento de la presencia de As en el suelo y en aguas subterráneas (Reimann et al., 2009). Esto hace suponer que al determinar una línea base de concentraciones iniciales de As disponible en los suelos de una región, así como su relación con los contenidos de arcilla, calcio y fósforo; además de la distribución espacial de este metaloide, se lograría una mejor comprensión del problema de arsenicismo, lo cual contribuiría en la formulación de estrategias de manejo agrícola y ambiental. En este sentido, las investigaciones enfocadas al conocimiento de la distribución espacial de este metaloide en la región Lagunera son escasas. Al respecto, los métodos de interpolación son procedimientos útiles para predecir valores desconocidos de cualquier dato (como elevaciones, precipitaciones, concentraciones químicas, niveles de ruido, etc.) de un punto geográfico. Los más utilizados son el método de ponderación de la distancia inversa (IDW por sus siglas en inglés) y el kriging ordinario (KO) (Li y Heap, 2011). El método IDW se considera como un método no-geoestadístico (Li y Heap, 2014) que emplea la Ley de Tobler (Tobler, 1970) para el cálculo de valores de sitios no muestreados con base en el promedio ponderado de los datos de sitios cercanos muestreados (Longley et al., 2010; Panagopoulos et al., 2006). La ponderación está dada por una función inversamente proporcional a la distancia entre el punto a ser estimado y el punto muestreado (De la Mora et al., 2004). En cambio, KO cuantifica la estructura espacial de los datos mediante el uso de variogramas llamados algunas veces semivariogramas debido a su similitud en el cálculo, y los predice mediante la interpolación usando estadística (Villatoro et al., 2008). Ambos modelos asumen que las predicciones son una 
combinación lineal de los datos (Schloeder et al., 2001). La efectividad de estos interpoladores ha sido comparada en la elaboración de mapas de distribución de diferentes características de los suelos, precipitación, y concentración de arsénico en aguas subterráneas, entre otros (Hernández y Ponce, 2006; Shi et al., 2009; Gordon et al., 2014). Sin embargo, la información relacionada con la comparación de mapas obtenidos a partir de $\mathrm{KO}$ e IDW en la distribución espacial de concentraciones de As edáfico en una región árida, es escasa. Los objetivos de este trabajo fueron determinar la concentración de As en la capa superficial $(0-30 \mathrm{~cm})$ de suelos del DR017, así como determinar su relación con el contenido de arcilla, fósforo y carbonatos de calcio de esos suelos y establecer su distribución especial utilizando los métodos de interpolación $\mathrm{KO}$ e IDW.

\section{MATERIALES Y MÉTODOS}

\section{Área de Estudio}

El área fue el Distrito de Riego 017 (DR017) ubicado en la Comarca Lagunera, en la parte suroeste del estado de Coahuila y el extremo noreste del estado de Durango en la zona norcentral de México, a $1100 \mathrm{~m}$ de altitud (Figura 1). El clima es un BW hw" (e') correspondiente a muy seco o desértico, semicálido con invierno fresco (García, 1988). La vegetación es semidesértica de tipo matorral desértico rosetófilo, sin erosión apreciable. Su geología es de clase sedimentaria del tipo caliza. Los suelos se clasifican dentro de los grupos Calcisoles, Gypsisoles, Feozem, Fluvisoles y Vertisoles (INEGI, 2006). Por otra parte, los campesinos de la región con base en su conocimiento tradicional, identifican cinco clases de tierra que nombra Blanca $(B l)$, Blanca Arenosa $(B a)$, Arenales $(A)$, Prietas $(P r)$ y Negras $(N)$, por las características que observan en los suelos como el color, la textura y la consistencia, que las relacionan con los cultivos y los rendimientos que obtienen (Segura-Castruita et al., 2012).

El trabajo se dividió en cinco etapas: 1) determinación de sitios de muestreo, 2) análisis de laboratorio, 3) generación de la cartografía de arsénico, 4) verificación de la precisión de mapas y 5) análisis de resultados.

Determinación de los sitios de muestreo. El número de sitios de muestreo o tamaño de muestra se obtuvo de acuerdo con Shabenberger y Pierce (2001), mediante la siguiente Ecuación 1:

$N=B \pi_{i}\left(1-\pi_{i}\right) / b_{i}^{2}$

donde: $N$ es el número de sitios de muestreo; $B$ es determinado por $j i$ cuadrada con un grado de libertad y $a-\alpha / c ; c$ representa el número de categorías en la clasificación, para el estudio es el número de clases de tierra (cinco $[B l, B a, P r, N$ y $A]$, más ninguna o algún otra clase de tierras diferente que indiquen los productores y no se tenga relacionada) como lo mencionan Segura-Castruita et al. (2012), $\alpha$ es el nivel de confianza $(95 \%) ; \pi_{i}$ es el más grande porcentaje para cada categoría o clase $(50 \%)$ y $b_{i}(30 \%)$ la probabilidad de que el resultado sea inferior al deseado. Por lo tanto, el número total de sitios o puntos de muestreo fue de 33 . La ubicación de los sitios de muestreo se realizó sobre una escena del DR017 en formato digital, de manera aleatoria (Francois et al., 2003), a través del módulo Hawth's Tools del software ArcGIS $10.1^{\circ}$ (SeguraCastruita et al., 2014) en la parte que corresponde al estado de Coahuila (Figura 1); aunque en la elección final de los sitios se tomó como criterio la accesibilidad

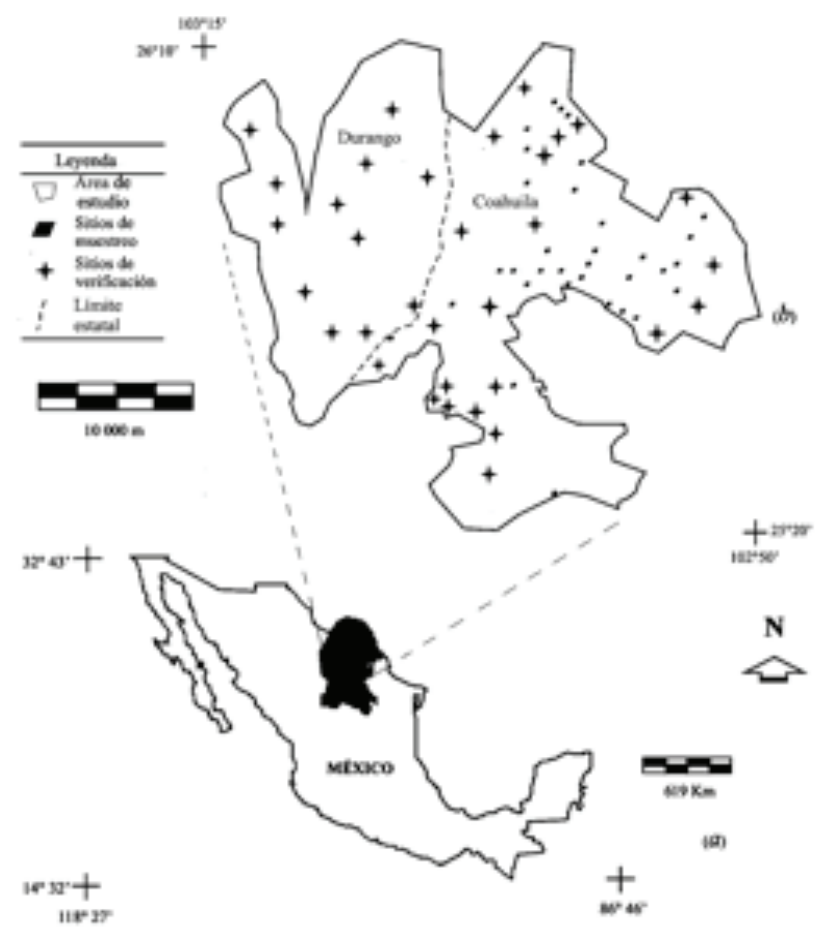

Figura 1. Localización del Distrito de Riego 017, área de estudio. 
a los lugares (carreteras pavimentadas, terracerías y brechas) con el apoyo de cartas topográficas del INEGI (1988). En cada sitio, una muestra simple de suelo ( $2 \mathrm{~kg}$, aproximadamente) de la capa superficial (0-30 $\mathrm{cm}$ de profundidad) fue colectada; es decir, un total de 33 muestras simples de suelo fueron obtenidas del área de estudio, que se trasladaron al laboratorio. Asimismo, cada sitio fue nuevamente georreferenciado con un GPS marca Garmin Etrex, ya que en algunos casos las coordenadas de los sitios no fueron exactas a las que originalmente se ubicaron en el área de estudio para el muestreo. Además, se realizó una entrevista a los productores dueños de las parcelas o lugares donde se encontraban los sitios de muestreo, con el fin de conocer la clase de tierra que correspondía, de acuerdo al manual para la cartografía de clases de tierras campesinas de Ortiz-Solorio et al. (1990), y relacionarlos a los resultados de contenidos de arsénico. Análisis de laboratorio. Las determinaciones físicas y químicas que se realizaron a los suelos fueron: análisis mecánico de partículas (método de la pipeta) para conocer el porcentaje de partículas de tamaño arcilla (r), carbonatos de calcio secundario (método de Horton y Newson), fósforo disponible (método de Olsen), y arsénico disponible (espectrometría de absorción atómica con generación de hidruros) (Norma Mexicana NOM-021-RECNAT-2000, SEMARNAT, 2002). Los análisis se realizaron por triplicado a cada uno de los suelos muestreados; donde cada suelo muestreado correspondió a un tratamiento y su determinación contó con tres repeticiones.

Generación de la cartografía de arsénico. La distribución espacial de las concentraciones de arsénico disponible en los suelos del DR017, se obtuvo al realizar la interpolación de los datos de contenidos de arsénico con respecto a su ubicación geográfica. Los métodos que se utilizaron fueron KO (Lin y Puls, 2008) e IDW, con un peso o poder en el clasificador de dos (Fortis et al., 2010), mediante los softwares ArcGIS $10.1^{\circ}$ y el IDRISI Kilimanjaro.

Verificación de la precisión de mapas. Los mapas de concentración de As (obtenidos con IDW y KO) fueron verificados en 33 puntos, distribuidos en forma aleatoria en cada mapa. El número de puntos o sitios fue el mismo que en el muestreo inicial, al considerar que ese fue el resultado del tamaño de muestra para el área de estudio. La distribución de estos se obtuvo nuevamente como se indicó en la sección de sitios de muestreo, con la salvedad que se realizó en toda el área que corresponde al distrito (Figura 1), para asegurar que no se tuviera la misma ubicación que los del muestreo inicial. En cada punto, la concentración de As fue registrado en una base de datos; asimismo, sus coordenadas fueron grabadas en un GPS marca Garmin, para acudir posteriormente a campo y realizar de nuevo la colecta de una muestra simple de suelo en cada sitio. Las muestras fueron trasladadas a laboratorio, donde se obtuvo el contenido de arsénico, mismo que se comparó con las concentraciones que indicaban los mapas temáticos (Segura-Castruita et al., 2014). Los resultados de la comparación sirvieron para elaborar una matriz de error con dos clases (acierto o error) para cada mapa (Segura-Castruita et al., 2012) con el fin de obtener la precisión de los mismos.

Análisis estadístico de los resultados. Se realizó una prueba de medias de los resultados de las características físicas y químicas mediante la prueba de Tukey $(P \leq 0.05)$. Además, relaciones lineales fueron establecidas entre las concentraciones de arsénico (variable dependiente) y el porcentaje de arcilla y $\mathrm{CaCO}_{3}$, así como con la concentración de fósforo, con el fin de determinar la influencia de éstas características en la presencia de arsénico. Asimismo, un análisis de varianza $(P \leq 0.05)$ fue realizado a estas regresiones. Por último, se llevó a cabo una prueba de bondad de ajuste a la precisión de los mapas, mediante una prueba de $j i$ cuadrada, con un grado de libertad y un nivel de significancia al $5 \%\left(X_{0.05}^{2}(1)\right)$, lo que da como resultado un valor crítico $X^{2}=3.84$, con el fin de contrastar que la precisión de los mapas es diferente, mediante la siguiente Ecuación 2:

$$
X^{2 *}=\sum_{i=1}^{k} \frac{\left(O_{i}-E_{i}\right)^{2}}{E_{i}}
$$

donde: $\mathrm{X}^{2 *}$ es el valor estadístico $j i$ cuadrada calculado, $O_{i}$ frecuencias observadas en cada clase (acierto, error) para cada mapa, $E_{i}$ frecuencias esperadas para cada clase (acierto, error) de cada mapa.

\section{RESULTADOS Y DISCUSIÓN}

\section{Arsénico en los Suelos del DR017 en la Comarca Lagunera}

La concentración de arsénico (As) disponible en los suelos de los diferentes sitios (Cuadro 1) presentó 
diferencias estadísticamente significativas $(P \leq 0.05)$. Lo anterior indica que la distribución de este metaloide en los suelos de la región no es uniforme; por lo que la concentración de este elemento podría estar relacionada con algunas características edáficas como el contenido de arcilla ( $\mathrm{r}), \mathrm{CaCO}_{3}$ y concentraciones de fósforo $(\mathrm{P})$ como indican Fixen y Grove (1990). Sin embargo, al relacionar el contenido de As con las características del suelo en estudio, se obtuvo una función lineal multivariada, que se presenta en la Ecuación 3:

$$
A s=0.314+0.010 r-0.026 \mathrm{CaCO}_{3}+0.001 P
$$

Cuadro 1. Concentraciones de arsénico y características edáficas de suelos del Distrito de Riego 017.

\begin{tabular}{|c|c|c|c|c|c|c|c|}
\hline \multirow[t]{2}{*}{ Muestra } & \multicolumn{2}{|c|}{ UTM } & As & $\mathrm{r}$ & $\mathrm{CaCO}_{3}$ & $P$ & Clase de tierra campesina \\
\hline & $\mathrm{X}$ & $\mathrm{Y}$ & $\mathrm{mg} \mathrm{kg}^{-1}$ & $-\cdots--0$ & $-\cdots$ & $\mathrm{mg} \mathrm{kg}^{-1}$ & \\
\hline 1 & 686051 & 2874816 & $1.89 \mathrm{a}^{\dagger}$ & 43.44 defg & $1.77 \mathrm{o}$ & 3.63 op & Prieta \\
\hline 2 & 687212 & 2872198 & $1.39 \mathrm{ab}$ & 39.44 ghi & $3.40 \mathrm{~lm}$ & $9.93 \mathrm{~g}$ & Negra \\
\hline 3 & 687259 & 2872365 & $1.49 \mathrm{ab}$ & 43.44 defg & $2.19 \mathrm{o}$ & $8.96 \mathrm{hi}$ & Prieta \\
\hline 4 & 686839 & 2873111 & $0.59 \mathrm{~cd}$ & $32.16 \mathrm{jk}$ & $4.00 \mathrm{k}$ & $7.31 \mathrm{kl}$ & Blanca arenosa \\
\hline 5 & 681076 & 2871083 & $0.48 \mathrm{~cd}$ & $39.28 \mathrm{ghi}$ & $6.41 \mathrm{i}$ & $7.12 \mathrm{klm}$ & Blanca arenosa \\
\hline 6 & 696338 & 2852715 & $0.20 \mathrm{~d}$ & 16.24 op & $3.40 \mathrm{~lm}$ & $6.64 \mathrm{~m}$ & Arenal \\
\hline 7 & 673694 & 2850376 & $0.35 \mathrm{~cd}$ & $29.12 \mathrm{kl}$ & $3.70 \mathrm{kl}$ & $7.02 \mathrm{klm}$ & Negra \\
\hline 8 & 700855 & 2851202 & $0.20 \mathrm{~d}$ & 40.00 fghi & $9.43 \mathrm{e}$ & $7.55 \mathrm{jk}$ & Prieta \\
\hline 9 & 684620 & 2847670 & $0.49 \mathrm{~cd}$ & 39.28 ghi & $4.60 \mathrm{j}$ & $7.02 \mathrm{klm}$ & Prieta \\
\hline 10 & 687674 & 2852029 & $0.07 \mathrm{~d}$ & $41.28 \mathrm{efgh}$ & $4.00 \mathrm{k}$ & $5.86 \mathrm{n}$ & Prieta \\
\hline 11 & 688921 & 2853733 & $0.13 \mathrm{~d}$ & 44.72 cdef & $6.41 \mathrm{i}$ & $6.64 \mathrm{~m}$ & Prieta \\
\hline 12 & 676174 & 2850402 & $0.07 \mathrm{~d}$ & $23.28 \mathrm{mn}$ & $4.60 \mathrm{j}$ & $4.21 \mathrm{o}$ & Blanca \\
\hline 13 & 677721 & 2850456 & $0.48 \mathrm{~cd}$ & 40.72 fghi & $4.00 \mathrm{k}$ & $6.73 \mathrm{~lm}$ & Prieta \\
\hline 14 & 694216 & 2849585 & $0.21 \mathrm{~d}$ & $35.84 \mathrm{ij}$ & $2.70 \mathrm{n}$ & $9.55 \mathrm{gh}$ & Negra \\
\hline 15 & 685195 & 2832691 & $0.20 \mathrm{~d}$ & 46.00 cde & $4.90 \mathrm{j}$ & $6.83 \mathrm{~lm}$ & Prieta \\
\hline 16 & 700030 & 2848494 & $0.34 \mathrm{~cd}$ & 19.28 no & $8.82 \mathrm{f}$ & $13.14 \mathrm{e}$ & Arenal \\
\hline 17 & 690303 & 2843282 & $0.35 \mathrm{~cd}$ & $26.00 \mathrm{~lm}$ & $12.02 \mathrm{c}$ & $9.06 \mathrm{hi}$ & Blanca arenosa \\
\hline 18 & 693812 & 2842386 & $0.35 \mathrm{~cd}$ & $8.00 \mathrm{q}$ & $3.10 \mathrm{mn}$ & $7.51 \mathrm{jk}$ & Arenal \\
\hline 19 & 681246 & 2853077 & $0.20 \mathrm{~d}$ & $43.28 \mathrm{defg}$ & $4.00 \mathrm{k}$ & $5.96 \mathrm{n}$ & Prieta \\
\hline 20 & 683474 & 2850527 & $0.48 \mathrm{~cd}$ & $55.02 \mathrm{~b}$ & $6.41 \mathrm{i}$ & $8.77 \mathrm{i}$ & Prieta \\
\hline 21 & 699187 & 2850281 & $1.04 \mathrm{bc}$ & 36.20 hij & $2.19 \mathrm{o}$ & $7.99 \mathrm{j}$ & Negra \\
\hline 22 & 677938 & 2849842 & $0.50 \mathrm{~cd}$ & 17.92 op & $9.60 \mathrm{e}$ & $3.23 \mathrm{p}$ & Arenal \\
\hline 23 & 677938 & 2868252 & $0.25 \mathrm{~d}$ & $29.20 \mathrm{kl}$ & $14.50 \mathrm{~b}$ & $9.26 \mathrm{hi}$ & Blanca \\
\hline 24 & 685137 & 2863144 & $0.75 \mathrm{~cd}$ & $33.20 \mathrm{jk}$ & $8.20 \mathrm{~g}$ & $55.46 \mathrm{a}$ & Blanca \\
\hline 25 & 689460 & 2858712 & $1.00 \mathrm{bc}$ & 43.92 cdefg & $10.30 \mathrm{~d}$ & 9.38 ghi & Prieta \\
\hline 26 & 704550 & 2859215 & $1.00 \mathrm{bc}$ & $59.20 \mathrm{~b}$ & $9.60 \mathrm{e}$ & $7.28 \mathrm{kl}$ & Prieta \\
\hline 27 & 702502 & 2856505 & $0.50 \mathrm{~cd}$ & $67.20 \mathrm{a}$ & $6.80 \mathrm{i}$ & $30.45 \mathrm{~b}$ & Prieta \\
\hline 28 & 667101 & 2845457 & $0.50 \mathrm{~cd}$ & $49.02 \mathrm{c}$ & $12.40 \mathrm{c}$ & $10.74 \mathrm{f}$ & Prieta \\
\hline 29 & 692305 & 2842062 & $0.10 \mathrm{~d}$ & $13.20 \mathrm{p}$ & $7.50 \mathrm{~h}$ & $21.46 \mathrm{c}$ & Arenal \\
\hline 30 & 684802 & 2849892 & $0.25 \mathrm{~d}$ & 40.20 fghi & $12.40 \mathrm{c}$ & $17.88 \mathrm{~d}$ & Prieta \\
\hline 31 & 681140 & 2851171 & $0.50 \mathrm{~cd}$ & $46.64 \mathrm{~cd}$ & $9.60 \mathrm{e}$ & $5.44 \mathrm{n}$ & Prieta \\
\hline 32 & 691197 & 2862134 & $0.25 \mathrm{~d}$ & $46.64 \mathrm{~cd}$ & $6.80 \mathrm{i}$ & $10.86 \mathrm{f}$ & Prieta \\
\hline 33 & 689989 & 2816649 & $0.50 \mathrm{~cd}$ & $48.64 \mathrm{c}$ & $20.79 a$ & $13.33 \mathrm{e}$ & Prieta \\
\hline
\end{tabular}


Los parámetros de esta ecuación tienen diferente significado, la ordenada al origen señala que, al no existir arcilla, carbonatos de calcio ni fósforo en el suelo, la concentración de arsénico disponible sería igual a $0.314 \mathrm{mg} \mathrm{kg}^{-1}$. Por otra parte, los coeficientes de regresión tienen diferentes tendencias (positivas y negativas) para cada variable independiente; es decir, cuando se incrementa en una unidad el porcentaje de arcilla o el contenido de fósforo en el suelo, la concentración de arsénico aumentará 0.010 y $0.001 \mathrm{mg} \mathrm{kg}^{-1}$, respectivamente. Diferentes investigaciones han demostrado que el contenido de arcilla tiene influencia en la retención de arsenatos en el suelo (Manning y Goldberg, 2007) como se encontró en este estudio. Por otra parte, la escasa relación entre el arsénico y el fósforo se debe principalmente al equilibrio que estos dos elementos guardan en la naturaleza (Liu et al., 2001), que se modifica al agregar fósforo como fertilizante (Hernández et al., 2013). En cambio, cuando el porcentaje de carbonato de calcio se incrementa en una unidad, el As disminuye $0.026 \mathrm{mg} \mathrm{kg}^{-1}$. Este comportamiento se puede explicar porque el ión $\mathrm{Ca}^{2+}$ promueve la adsorción de arsenatos y carbonatos en el suelo; también, puede alterar la retención/movilización de As en el suelo (Stachowicz et al., 2008). Sin embargo, la ecuación tiene una $\mathrm{R}^{2}$ igual que 0.115 , lo que significa que sólo explica el $11.5 \%$ del fenómeno. Es decir, las características edáficas seleccionadas en combinación, no ayudan a esclarecer el comportamiento de la concentración de arsénico en los suelos del DR017.

No obstante, el análisis de varianza de la regresión (Cuadro2) indica que el modelo estimadoes significativo $(P \leq 0.05)$ ya que la probabilidad de rechazo fue igual a 0.009 ; además, la probabilidad de rechazo $(P)$ y la $F$ calculada de las variables independientes es diferente, así como el error estandarizado de los coeficientes (EEC), lo cual indica que considerar en la regresión únicamente a la ry a los $\mathrm{CaCO}_{3}$ por separado sería más apropiado para tratar de explicar el comportamiento del arsénico en estos suelos.

$\mathrm{Al}$ analizar la agrupación de medias de los valores más altos de arsénico (Tukey, $P \leq 0.05$ ) en los suelos del área de estudio, cuatro sitios presentaron la mayor concentración (Cuadro 1); estos sitios se ubicaron al norte del DR017 y correspondieron a las clases de tierra prieta y negra. Al establecer nuevamente la relación entre la concentración de As y el contenido de arcilla, de acuerdo con lo mencionado anteriormente, así como de un sitio más (blanca arenosa) que se encontraba próximo a los tres anteriores, con el fin de captar la variabilidad en esa área, se tuvo el modelo que se muestra en la Figura 2. Este modelo es estadísticamente significativo $(P \leq 0.05)$ de tal manera que la ecuación de regresión puede explicar $85.87 \%$ ( $\mathrm{R}^{2}$ ajustada) de la variación del As en el rango de contenidos de arcilla que fluctúan de 32.16 a $43.44 \%$. En este sentido, la ecuación podría utilizarse para predecir contenidos de As, en el rango antes mencionado.

Este comportamiento se ha reportado en suelos de California, donde se explica que los suelos con mayor contenido de arcilla tienen una mayor capacidad para retener arsénico (Manning y Goldberg, 2007); sin embargo, no se establece el tipo de arcilla que está influyendo en este fenómeno. Por otra parte, en la presente investigación se constató que los cultivos que se encuentran en suelos del área norte del DR017, son irrigados con agua que se extrae del subsuelo.

Cuadro 2. Análisis de varianza de la regresión multivariada entre la concentración de arsénico y el contenido de arcilla, carbonatos de calcio $\left(\mathrm{CaCO}_{3}\right)$ y fósforo $(\mathrm{P})$.

\begin{tabular}{|c|c|c|c|c|c|c|c|}
\hline Parámetro & $\mathrm{GL}^{\dagger}$ & SC ajustado & $\mathrm{CR}$ & EEC & $\mathrm{T}$ & Valor F & $P$ \\
\hline Regresión & 3 & 2.42 & & & & 4.05 & 0.009 \\
\hline Constante & & & 0.314 & & 2.07 & & 0.041 \\
\hline $\mathrm{r}$ & 1 & 1.59 & 0.01 & 1.287 & 2.83 & 7.98 & 0.006 \\
\hline $\mathrm{CaCO}_{3}$ & 1 & 1.13 & -0.026 & 1.097 & -2.38 & 5.67 & 0.015 \\
\hline$P$ & 1 & 0.32 & 0.002 & 0.032 & 0.4 & 0.16 & 0.72 \\
\hline Error & 95 & 18.92 & & & & & \\
\hline Total & 98 & 21.34 & & & & & \\
\hline
\end{tabular}

$\mathrm{GL}=$ grados de libertad; $\mathrm{SC}=$ suma de cuadrados; $\mathrm{CR}=$ coeficiente de regresión; $\mathrm{EEC}=$ error estandarizado de los coeficientes; $\mathrm{T}=\mathrm{t}$ de tablas; $F=$ estadística de probabilidad; $P=$ probabilidad de rechazo menor o igual a 0.05 . 


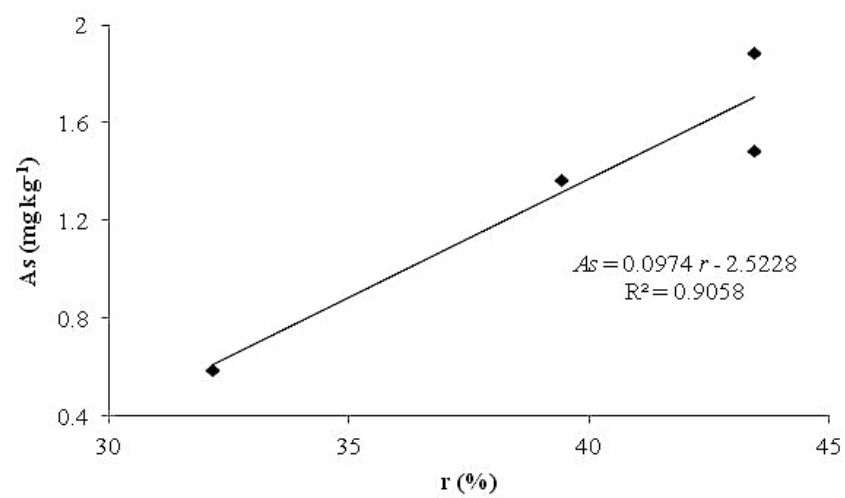

Figura 2. Relación de arsénico con respecto en el contenido de arcilla en suelos del norte del Distrito de Riego 017.

Aguilar-Muñiz et al. (2012) indican que, las aguas de los mantos acuíferos de esta zona tienen contenidos de As $>0.25 \mathrm{mg} \mathrm{L}^{-1}$. Lo anterior sugiere que la presencia y tipo de arcilla, así como la calidad del agua que se utiliza en su manejo, influyen en la distribución y disponibilidad del arsénico en estos suelos.

En cambio, el modelo de la presencia de As con el contenido de $\mathrm{CaCO}_{3}$, no es estadísticamente significativo $(P \leq 0.05)$. No obstante, este modelo muestra una tendencia negativa (Figura 3); es decir, la concentración de arsénico disminuye $0.4770 \mathrm{mg} \mathrm{kg}^{-1}$ cuando el porcentaje de $\mathrm{CaCO}_{3}$ se incrementa una unidad. De acuerdo con Yokoyama et al. (2012), el pH alcalino propicia la unión del metaloide a los $\mathrm{CaCO}_{3}$. Los $\mathrm{pH}$ de los suelos del área de estudio son de neutros a alcalinos, lo que ayuda a explicar el comportamiento del As respecto al $\mathrm{CaCO}_{3}$.

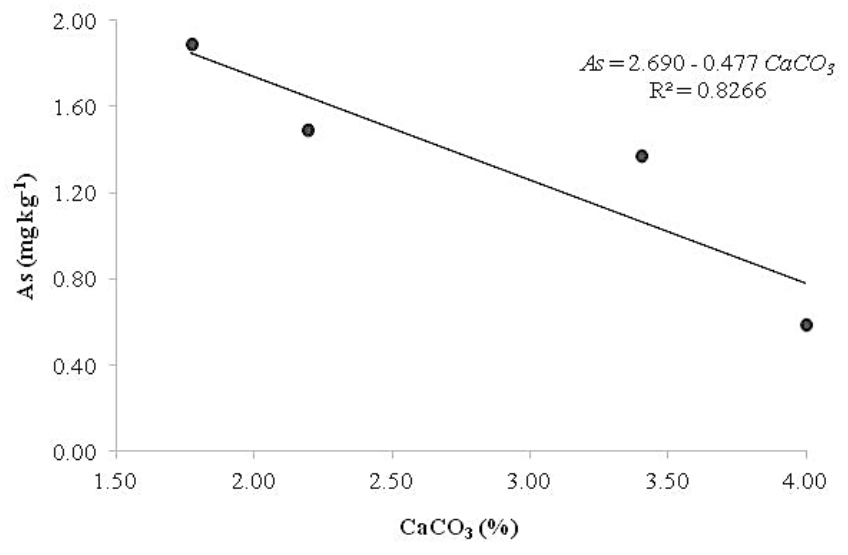

Figura 3. Relación de $\mathrm{CaCO}_{3}$ con respecto al contenido de As en el suelo del norte del Distrito de Riego 017.

Lo anterior permite deducir la presencia de arsenato de calcio o de un mineral con As (en combinación con el carbonato de calcio) en los suelos de este estudio. En otras palabras, procesos pedogenéticos que involucran As y $\mathrm{CaCO}_{3}$ de manera natural, permiten que el As en la solución del suelo tenga baja disponibilidad en presencia de $\mathrm{CaCO}_{3}$; pero en este estudio no se realizaron pruebas al respecto.

\section{Precisión de los Mapas de Concentración de Arsénico}

Los mapas de concentración de arsénico (Figura 4) muestran cinco clases de contenido de arsénico disponible. En los mapas se puede observar que

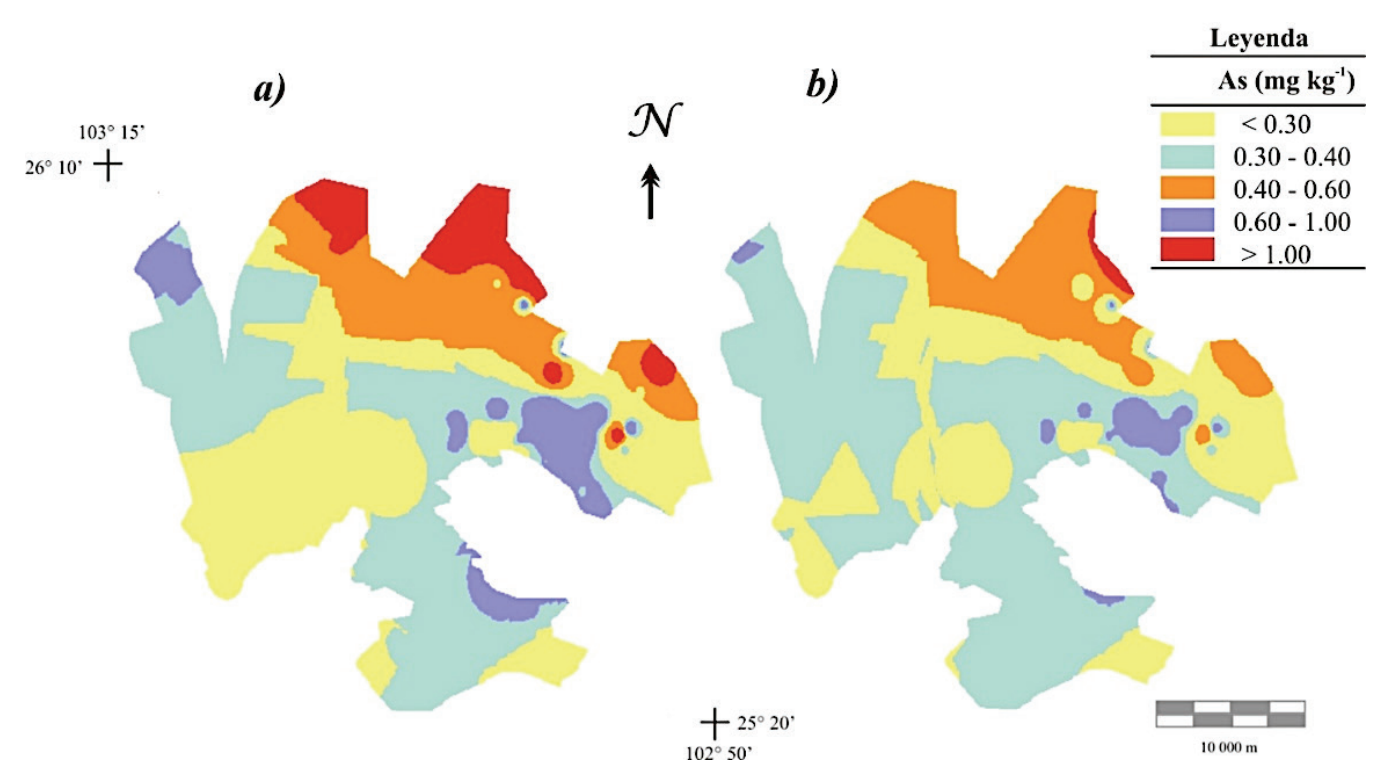

Figura 4. Mapas de distribución espacial de arsénico en el Distrito de Riego 017 (a) Método KO; (b) Método IDW. 
la mayor concentración de arsénico se encuentra al norte del DR017, lo que está relacionado con las mayores concentraciones de As y los altos contenidos de arcilla, como se explicó anteriormente.

El mapa obtenido con el método KO (Figura 4a) muestra una superficie de 21387.83 ha (Cuadro 3) con altos contenidos de As $\left(>1.0 \mathrm{mg} \mathrm{kg}^{-1}\right)$, mayor que la del mapa elaborado con IDW (Figura 4b); mientras que, con el IDW la más grande superficie (154 924.01 ha) fue la que presenta contenidos de 0.30 a $0.40 \mathrm{mg} \mathrm{kg}^{-1}$, que cubre un $51.26 \%$ del área total del distrito (302 206.51 ha). En cambio, cuando el mapa se obtuvo con $\mathrm{KO}$, los contenidos $<0.30 \mathrm{mg} \mathrm{kg}^{-1}$ ocuparon un área de 107510.48 ha, que corresponde a $35.57 \%$ del área total del DR017.

En cuanto a la precisión de los mapas temáticos obtenidos con IDW y KO no fue similar (Cuadro 4). El mapa con mayor precisión (75.7\%) fue el que se obtuvo con KO. Lo anterior se constató con el análisis de bondad de ajuste de las distribuciones de los resultados de la precisión de los mapas, donde el resultado fue menor $\left(X^{2 *}=1.32\right)$ que el valor crítico, por lo tanto, se acepta que los mapas tienen una precisión diferente. Al analizar el contraste de cada mapa se encontró que con KO se tuvo el mayor ajuste $\left(X^{2^{*}}{ }_{K O}=0.48\right)$ menor que 0.86 de IDW y por ende $\mathrm{KO}$ es más eficiente en la predicción de la presencia de As en la región. Este resultado es acorde con lo reportado por otros investigadores, quienes mencionan que el interpolador más popular y rápido es Kriging (Isaaks y Srivastava, 1989; Xie et al., 2011).

Las diferencias entre los interpoladores se deben a que el IDW considera relaciones de distancia simple (Li y Heap, 2014), dando como resultados valores entre el mínimo y máximo de los datos observados ( $\mathrm{Li}$ y Heap, 2008), lo cual depende de la configuración

Cuadro 3. Áreas de las clases de concentraciones de arsénico disponible edáfico del Distrito de Riego 017.

\begin{tabular}{|c|c|c|}
\hline As & $\mathrm{KO}$ & IDW \\
\hline $\mathrm{mg} \mathrm{kg}^{-1}$ & $\ldots \ldots-\ldots$ & $\ldots \ldots$ \\
\hline$<0.30$ & 107510.48 & 88721.56 \\
\hline $0.30-0.40$ & 103572.91 & 154924.01 \\
\hline $0.40-0.60$ & 45030.31 & 47668.10 \\
\hline $0.60-1.00$ & 24704.98 & 9363.06 \\
\hline$>1.00$ & 21387.83 & 1529.79 \\
\hline
\end{tabular}

Cuadro 4. Matriz de precisión de mapas de concentración de arsénico edáfico del Distrito de Riego 017.

\begin{tabular}{lcccc}
\hline Mapa & $\mathrm{N}$ & Acierto & Error & Precisión \\
\hline KO & 33 & 25 & 8 & $\%$ \\
IDW & 33 & 20 & 10 & 60.6 \\
\hline N = tamaño de la muestra. & & &
\end{tabular}

del interpolador o algoritmo clasificador; es decir, el exponente que determina el peso o poder asignado a cada una de las observaciones. Al respecto, Cruz et al. (2010) afirmaron que el mejor poder es ocho. En este estudio el peso o poder que se utilizó fue dos, lo que probablemente provocó que la precisión del interpolador disminuyera. En cambio, KO considera la cercanía de la vecindad de los diferentes puntos que lo rodean (Xie et al., 2011), así como minimiza la varianza de los valores de los puntos observados, lo que le permite obtener resultados fuera del rango observado (Li y Heap, 2014) e incrementa su eficiencia (Villatoro et al., 2008). No obstante, en este estudio el área de muestreo para la verificación del mapa al ser mayor que la explorada inicialmente en el estudio, pudo limitar la precisión del interpolador Kriging.

\section{CONCLUSIONES}

- La capa superficial de los suelos $(0-30 \mathrm{~cm}$ de profundidad) del Distrito de Riego 017 presentó concentraciones de arsénico (As) que fluctúan de 0.07 a $1.89 \mathrm{mg} \mathrm{kg}^{-1}$. El arsénico disponible en estos suelos depende de los contenidos de arcilla y carbonatos de calcio, principalmente; mientras que el fósforo disponible en estos suelos no influye en la presencia de As, ya que se encuentran en equilibrio. Las mayores concentraciones de este metaloide (1.39-1.89 $\mathrm{mg} \mathrm{kg}^{-1}$ ) están asociados a altos contenidos de arcilla de los suelos (39.44 a 43.44\%), correspondientes a tierras que los campesinos clasifican como negras y prietas, que se encontraban en la parte norte del Distrito de Riego 017 de la región de Coahuila; la relación entre las concentraciones de As disponible y contenidos de arcilla fue alta $\left(\mathrm{R}^{2}=0.9058\right)$ en los suelos de este lugar. - La distribución espacial del arsénico disponible en el distrito de riego presentó concentraciones que se incrementa en un sentido de sur a norte. El mapa obtenido con el interpolador Kriging ordinario mostró 
una precisión (75.7\%) mayor que el derivado del método inverso de la distancia. Aun cuando este resultado es aceptable, es necesario considerar que la distancia entre el límite del área de estudio y los puntos de muestreo para la verificación, fue amplia, lo que probablemente limitó la precisión del interpolador. Por tanto, se requeriría incrementar el espacio de distribución de los sitios de muestreo, a fin de aumentar la precisión del interpolador Kriging en la región.

\section{AGRADECIMIENTOS}

A el Tecnológico Nacional de México, antes Dirección General de Educación Superior Tecnológica, que financió este trabajo mediante el Proyecto con Clave 5168.13-P.

\section{LITERATURA CITADA}

Aguilar-Muñiz., A. U., F. Valdés P. y G. García V. 2012. Efectos estacionales en los niveles de arsénico de pozos urbanos de la Comarca Lagunera. IB: 12-47. Ibersensor 7: 132-133.

Alam, M. G., M. Tokunaga, and S. Stagnitti. 2007. Removal of arsenic from contaminated soils using different salt extractants. J. Environ. Sci. Heath A. 42: 447-451.

Anguiano C., J., J. J. Alcántar R., J. A. Ruiz C., I. J. González A., I. Vizcaíno V., J. R. Regalado R. y C. De la Mora O. 2003. Recursos edafo-climáticos para la planeación del sector productivo en el estado de Michoacán. Libro Técnico No. 1. INIFAP-CIRPAC. Uruapan, Michoacán, México.

Bissen, M. and F. H. Frimmel. 2003. Arsenic - a review. Part II: oxidation of arsenic and its removal in water treatment. Acta Hydroch. Hydrobiol. 31: 97-107.

Castruita Á., L., A. M. García L., J. E. Camporredondo S., C. F. Sifuentes B. y M. Garza G. 2011. Estudio cinético de la remoción de arsénico en un reactor electroquímico tipo filtro prensa. Prospectiva 9: 7-12.

Cruz C., G., C. A. Ortiz S., E. Ojeda T., J. F. Martínez M., E. D. Sotelo R., and A. L. Licona V. 2010. Evaluation of four digital classifiers for automated cartography of local soil classes based on reflectance and elevation in Mexico. Int. J. Remote Sens. 31: 665-679.

De la Mora O., C., J. G. Flores G., J. A. Ruiz C. y J. García V. 2004. Modelaje estocástico de la variabilidad espacial de la calidad de agua en un ecosistema lacustre. Rev. Int. Contam. Ambient. 20: 99-108.

Donghee, K., K. Kyu-Sun, K. Seongkwon, C. Youngmin, and L. Woojin. 2012. Assessment of geotechnical variability of Songdo silty clay. Eng. Geol. 133-134: 1-8.

Fitz, W. J. and W. W. Wenzel. 2002. Arsenic transformations in the soil/rhizosphere/plant system: fundamentals and potential application to phytoremediation. J. Biotechnol. 99: 259-278.

Fixen, P. E. and J. H. Grove. 1990. Testing soils for phosphorus. pp. 141-180. In: R. L. Westerman (ed.). Soil testing and plant analysis. SSSA Book Ser. 3. Madison, WI, USA.
Flores L., H. E., K. F. Byerly M., J. J. Aceves R. y J. A. Ruiz C. 2002. Diagnóstico del sistema de producción de agave con énfasis en problemas fitosanitarios. pp. 63-95. In: H. E. Flores López (ed.). Análisis agroecológico del Agave tequilana Weber var. Azul con énfasis en problemas fitosanitarios en Jalisco. INIFAP-CIRPAC-C.E. Altos de Jalisco. Tepatitlán, Jalisco, México.

Fortis H., M., A. Huerta G., M. A. Segura C., J. L. García H., J. A. Leos R., A. García S. y R. Valdez C. 2010. Validación de cuatro modelos de interpolación para cartografiar nitrato $\mathrm{y}$ amonio en suelo. Terra Latinoamericana 28: 371-379.

Francois M., J., J. R. Díaz G. y A. Pérez V. 2003. Evaluación de la confiabilidad temática de mapas o de imágenes clasificadas: Una revisión. Investig. Geográf. 51: 53-72.

García, E. 1988. Modificaciones al sistema de clasificación climática de Köppen. Instituto de Geografía, Universidad Nacional Autónoma de México. México, D. F.

Gordon, G., S. Mattevada, and S. E. O'Bryant. 2014. Comparison of the accuracy of kriging and IDW interpolations in estimating groundwater arsenic concentrations in Texas. Environ. Res. 130: 59-69.

Hernández S., J. L. and R. Ponce H. 2006. Mapping the spatial variability of plant diversity in a tropical forest: Comparison of spatial interpolation methods. Environ. Monit. Assess. 117: 307-334.

Hernández O., G., M. A. Segura C., L. C. Álvarez G., R. A. Aldaco N., M. Fortis H. y G. González C. 2013. Comportamiento del arsénico en suelos de la Región Lagunera de Coahuila, México. Terra Latinoamericana 31: 295-303.

INEGI (Instituto Nacional de Estadística, Geografía e Informática). 1988. Atlas nacional del medio físico. Aguascalientes, México.

INEGI (Instituto Nacional de Estadística Geografía e Informática). 2006. Conjunto de datos edafológicos alfanuméricos escala 1:250 000 Serie I, Continuo Nacional. INEGI. Aguascalientes, México.

Isaaks, E. H. and R. M. Srivastava. 1989. An introduction to applied geostatistics. Oxford University Press Nueva York, NY, USA. ISBN: 9780195050134.

Kolat, Ç., R. Ulusay, and M. Lütfi Süzen. 2012. Development of geotechnical microzonation model for Yenisehir (Bursa, Turkey) located at a seismically active region. Eng. Geol. 127: 36-53.

Li, J. and A. D. Heap. 2008. A review of spatial interpolation methods for environmental scientists. Geoscience Australia. ISSN 1448-2177

Li, J. and A. D. Heap. 2011. A review of comparative studies of spatial interpolation methods in environmental sciences: Performance and impact factors. Ecol. Informat. 6: 228-241.

Li, J. and A. D. Heap. 2014. Spatial interpolation methods applied in the environmental sciences: A review. Environ. Modell. Soft. 53: 173-189.

Lin, Y. P. 2008. Simulating spatial distributions, variability and uncertainty of soil arsenic by geostatistical simulations in geographic information systems. Open Environ. Sci. 2: 26-33.

Lin, Z. and R. W. Puls.2008. Adsorption, desorption and oxidation of arsenic affected by clay minerals and aging processes. Environ. Geol. 39: 753-759.

Liu, F., A. de Cristofaro, and A. Violante. 2001. Effect of pH, phosphate and oxalate on the adsorption/desorption of arsenate on/from goethite. Soil Sci. 166: 197-208. 
Longley, P., M. Goodchild, D. Maguire, and D. Rhind. 2010. Geographic information systems and science. John Wiley and Sons. Hoboken, NJ, USA.

Manning, B. and S. Goldberg. 2007. Arsenic (III) and arsenic (V) adsorption on three California soils. Soil Sci. 162: 886-895.

Ortiz-Solorio, C. A., D. Pájaro-Huertas y V. M. Ordaz-Chaparro. 1990. Manual para la cartografía de clases de tierras campesinas. Serie Cuadernos de Edafología 15. Centro de Edafología, Colegio de Postgraduados. Montecillo, Estado de México, México.

Panagopoulos, T., J. Jesus, M. D. C. Antunes, and J. Beltrao. 2006. Analysis of spatial interpolation for optimising management of a salinized field cultivated with lettuce. Eur. J. Agron. 24: 1-10.

Reimann, C., J. Matschullat, M. Birke, and R. Salminen. 2009. Arsenic distribution in the environment: The effects of scale. Appl. Geochem. 24: 1147-1167.

Schloeder, C. A., N. E. Zimmerman, and M. J. Jacobs. 2001. Comparison of methods for interpolating soil properties using limited data. Soil Sci. Soc. Am. J. 65: 470-479.

Segura-Castruita, M. A., L. Martínez C., E. García B., A. Huerta G., J. L. García H., M. Fortiz H., J. A. Orozco V., and P. Preciado R. 2012. Localization of local soil classes in an arid region of Mexico, using satellite imagery. Int. J. Remote Sens. 33: 184-197.

Segura-Castruita, M. A. 2014. Revisión: The soils of México. Bol. Soc. Geol. Mex. 66: 223-224.

Segura-Castruita., M. A., A. Huerta G., M. Fortis H., J. A. Montemayor T., L. Martínez C. y P. Yescas C. 2014. Cartografía de la probabilidad de ocurrencia de Atriplex canescens en una región árida de México. Agrociencia 48: 639-652.
SEMARNAT (Secretaría de Medio Ambiente y Recursos Naturales). 2002. Norma mexicana para análisis de laboratorio para clasificación de suelos. NOM-21-RECNAT-2000. México, D. F.

Shabenberger, O. and F. J. Pierce. 2001. Contemporary statistical models for the plants and soil science. CRC Press LLC. Boca Raton, FL, USA.

Shi, W., J. Liu, Z. Du, Y. Song, Ch. Chen, and T. Yue. 2009. Surface modelling of soil pH. Geoderma 150: 113-119.

Stachowicz, M., T. Hiemstra, and W. H. van Riemsdijk. 2008. Multi-competitive interaction of As (III) and As(V) oxyanions with $\mathrm{Ca}^{2+}, \mathrm{Mg}^{2+}, \mathrm{PO}_{3-4}$, and $\mathrm{CO}_{2-3}$ ions on goethite. J. Coll. Interf. Sci. 320: 400-414.

Tobler, W. R. 1970. A computer movie simulating urban growth in the Detroit region. Econ. Geogr. 46: 234-240.

Villatoro, M., C. Henríquez y F. Sancho. 2008. Comparación de los interpoladores IDW y Kriging en la variación espacial de pH, Ca, CICE y P del suelo. Agron. Costarricense 32: 95-105.

Violante, A. and M. Pigna. 2002. Competitive sorption of arsenate and phosphate on different clay minerals and soils. Soil Sci. Soc. Ame. J. 66: 1788-1796.

Xie, Y., T. Chen, M. Lei, J. Yang, Q. Guo, B. Song, and Z. Zhou. 2011. Spatial distribution of soil heavy metal pollution estimated by different interpolation methods: Accuracy and uncertainty analysis. Chemosphere 82: 468-476.

Yokoyama, Y., K. Tanaka, and Y. Takahashi. 2012. Differences in the immobilization of arsenite and arsenate by calcite. Geochim. Cosmochim. Acta 91: 202-219.

Zhao, F. J., J. F. Ma, A. A. Meharg, and S. P. McGrath. 2009. Arsenic uptake and metabolism in plants. New Phythol. 181: 777-794. 